\title{
Demand side management using the internet of energy based on LoRaWAN technology
}

\author{
Om-Kolsoom Shahryari \\ Department of Computer Engineering, Sanandaj Branch \\ Islamic Azad University \\ Sanandaj, Iran \\ shahryari.k@iausdj.ac.ir
}

\author{
Amjad Anvari-Moghaddam \\ Department of Energy technology \\ Aalborg University \\ Aalborg, Denmark \\ aam@et.aau.dk
}

\author{
Shadi Shahryari \\ Department of Computer Engineering \\ University of Kurdistan \\ Sanandaj, Iran \\ shadi.sharyari2@gmail.com
}

\begin{abstract}
The smart grid, as a communication network, allows numerous connected devices such as sensors, relays and actuators to interact and cooperate with each other. An Internet-based solution for electricity that provides bidirectional flow of information and power is internet of energy (IoE) which is an extension of smart grid concept. A large number of connected devices and the huge amount of data generated by IoE and issues related to data transmission, process and storage, force IoE to be integrated by cloud computing. Furthermore, in order to enhance the performance and reduce the volume of transmitted data and process information in an acceptable time, fog computing is suggested as a layer between IoE layer and cloud layer. This layer is used as a local processing level that leads to reduction in data transmissions to the cloud. So, it can save energy consumption used by IoE devices to transmit data into cloud because of a long range, low power, wide area and low bit rate wireless telecommunication system which is called LoRaWAN. All devices in fog domain are connected by long range wide area network (LoRa) into a smart gateway. The gateway which bridges fog domain and cloud, is introduced for scheduling devices/appliances by creating a priority queue which can perform demand side management dynamically. The queue is affected by not only the consumer importance but also the consumer policies and the status of energy resources.
\end{abstract}

Keywords: Internet of Things, Internet of Energy, fog computing, cloud computing, LoRaWAN, microgrid, demand side management.

\section{INTRODUCTION}

The Internet of things (IoT) is considered as the next phase in the evolution of the Internet. Internet of Things has been identified as one of the emerging technologies in IT as noted in Gartner's IT Hype Cycle. It has been forecasted that IoT will take 5-10 years for market adoption [1]. IoT devices rapidly grow and are used in industries and various domains. IoT connects people, machines and devices to each other and to the internet and enables bidirectional information transmission and realtime decisions [2]. Two key factors which affect growth rate of IoT, are reduction in size and cost of IoT devices. IoT can play an essential role in applications related to private users such as smart homes, wearable tools, learning ad applications related to business users such as automation and industrial manufacturing, intelligent transportation, smart city, smart grid and e-health [3]. IoT has huge potentialities for developing new intelligent applications in nearly every field. The various applications can be grouped in three major domains: (A) industrial domain, (B) smart city domain, and (C) health well-being domain. Each domain is not isolated from the others but it is partially overlapped since some applications are shared. Fig. 1 shows the subdivision in the aforementioned domains and provides a nonexhaustive list of IoT applications for each of them [4]. Bob Metcalfe, inventor of Ethernet and expert in technology domain, says that: "over the past 63 years it has been tried to provide clean and cheap information for world by Internet; however in the next 63 years the need of world for cheap and clean energy should be fulfilled by Enernet" [5]. Enernet is the idea that we can learn from the history of the Internet "how to meet accelerating world needs for cheap and clean energy". Private industries and governments are accelerating the promotion of using innovations for smart grid applications. Some investments should be done in this domain to provide an agile platform for power generation and distribution in smart energy system that allow customers save money by scheduling the proper time for effective device usages [6]. Historical Data shows that a large part of energy has been generated from fossil fuels (in 2011, 82\%) [7]. The widespread use of fossil fuels has damaging and undesirable environmental impacts such as global warming caused by $\mathrm{CO} 2$ emissions from them [8]. Also, there is another political challenge, energy insecurity which denotes that most imported oil comes from politically volatile regions [9]. So, a sustainable approach to face this problem is to replace fossil fuels by renewable energy resources [10]. Therefore, there is an increasing pervasive focus on renewable energy systems to replace fossil fuels and how to meet future demand for electricity. The International Energy Agency in [11] says that the share of renewable energy in global power generation will rise up to $26 \%$ by 2020 . Using renewable energy resources such as solar and wind generation for homes and buildings presents a new challenge for balancing energy on the power grid because they do not supply constant power and have stochastic behavior over the time [6],[12]-[13]. There are some challenges regarding the wide spread adoption of renewable energy such as ability 
to store and control the wide variety of different energy resources which typically require complex control of diverse and distributed energy sources and storage to meet demand [9]. Smart grid is an intelligent distributed infrastructure that manages energy needs in a sustainable, reliable and economic manner with the help of reliable high-speed communication networks for monitoring and control [14]-[15]. Convergence of smart grid and IoT is called Internet of Energy (IoE) and can be seen as an extension of the smart grid concept [16]. The goal of $\mathrm{IoE}$ is to provide a robust system for energy exchange between prosumers. One of the issues raised in IoE is Demand Side Management (DSM). DSM refers to techniques and technologies to planning, monitoring and implementing activities and behaviors of electrical utility which the ultimate goal is encourage consumers to optimize their energy usage.

Distributed and intermittent energy production and storage needs to be monitored and controlled intelligently via Internet. IoE will allow energy exchange between a wide variety of sources and loads, including renewable energy sources, distributed energy storage, plug-in electric vehicles, domestic and industrial prosumers, etc. To overcome increasing complexity and enormous amount of data that generated by a large number of devices such as smart meters, sensors, actuators and relays, which has to be stored, processed, and accessed, the powerful processing resources are required which can be provide by cloud computing. The combination of cloud computing and IoT for creating IoE platform which can enable ubiquitous sensing services and allow the sensing data to be stored and used intelligently for smart monitoring and powerful processing of sensing data streams. However, increasing the number of IoE devices leads to an increase in response time and latency in cloud computing which causes deviations from the time requirements for some delay-sensitive devices and applications [17]. To conquer these challenges, the fog computing has been suggested in [18]. Fog computing pulls the cloud computing to the edge of the network and allows data to be preprocessed whenever latency limitation is required [19] and leads to an increase in interoperability, scalability, consistency and better connectivity between smart devices [17]. The most important features of Fog area which can be mentioned are: low latency and location awareness, wide-spread geographical distribution, mobility, very large number of nodes, predominant role of wireless access, strong presence of streaming and real-time applications and heterogeneity [19]. IoT faces some challenges such as stringent latency requirements, network bandwidth constraints, resource-constrained devices, cyber- physical systems that connected to IoT, uninterrupted services with intermittent connectivity to the cloud, new security challenges, keeping security credentials and software up to date on a large number of devices, protecting resource-constrained devices, assessing the security status of large distributed systems in a trustworthy manner and

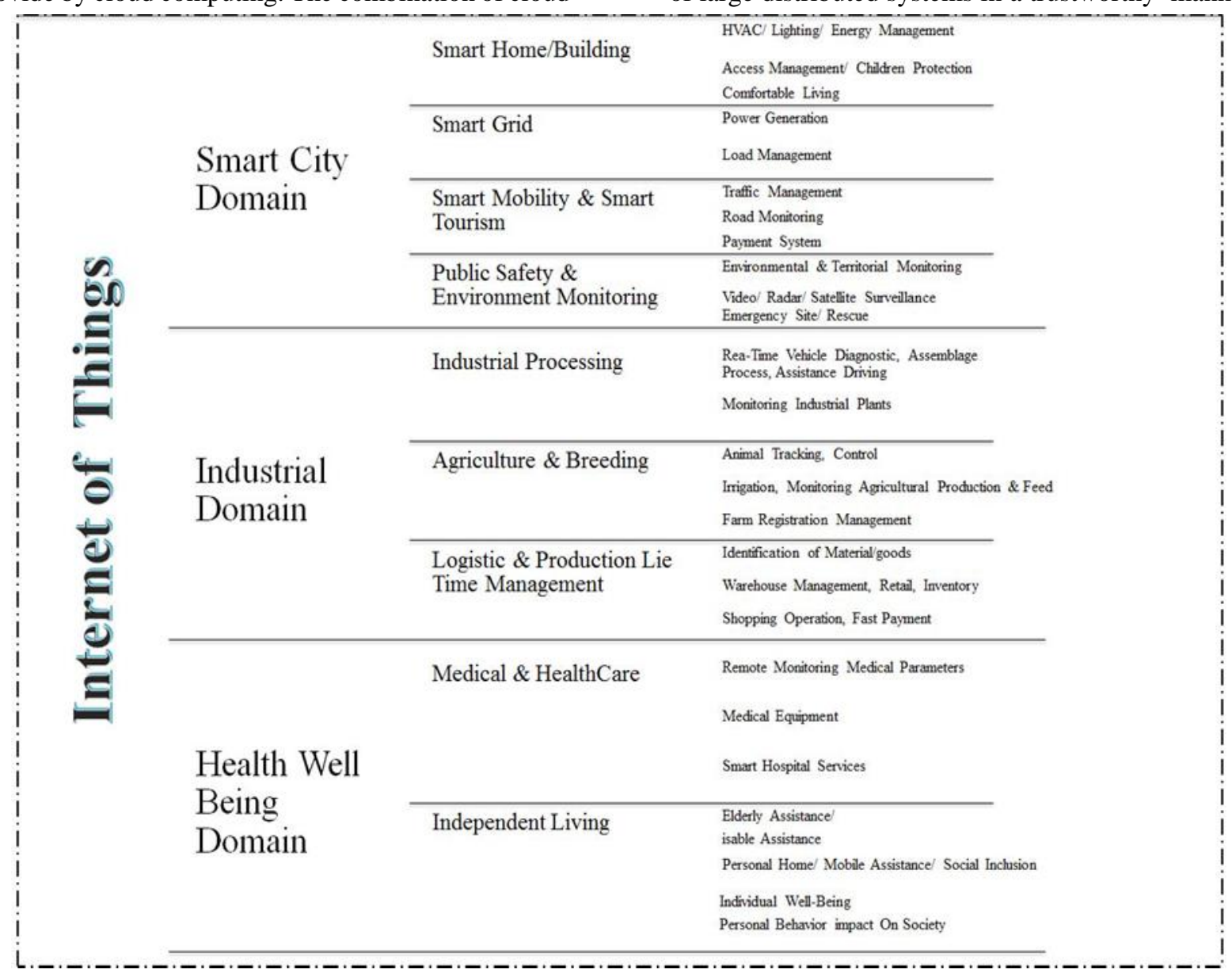

Figure 1 List of IoT applications 
responding to security compromises without causing intolerable disruptions which cannot be satisfied by cloud computing. Fog is a computing and networking architecture that distributes computing, control, storages and networking functions closer to end-user devices [20]. In our previous work [21], an effective platform for IoE based on combining two technologies: cloud computing and fog computing was proposed. In order to more reduction in energy consumption by devices, the LowPower Wide-Area Network (LPWAN) can be used which is a type of wireless telecommunication wide area network designed to allow long range communications at a low bit rate among things (connected objects), such as sensors operated on a battery [22]. LoRa is one of the LPWAN protocols which is a new wireless protocol designed specifically for long-range, low-power communications. LoRa stands for Long Range Radio and is mainly targeted for M2M and IoT networks [23].

Thus, this paper elaborates on presenting an effective platform for demand side management which integrated IoT, cloud computing, fog computing and uses the LoRaWAN technology in fog domain.

The paper is organized as follow: section 2 describes the cloud computing briefly. In section 3 , the fog computing is presented. In section 4 the LoRaWAN technology is discussed. Then, the fog computing application for smart grid is discussed in section 5. Finally, section 6 draws the conclusions.

\section{CLOUD COMPUTING}

Cloud Computing is an internet based infrastructure which enables on-demand access to shared configurable resources (applications, services, storages, servers, network and etc.) via Internet. The main features of cloud computing are: self-service, broadband network access, reliability, manageability, resource pooling, rapid elasticity, and measured service. Cloud Computing considered everything as a service (XaaS). Infrastructure as a service (IaaS), Platform as a service (PaaS) and Software as a service (SaaS) are three types of cloud services that provided to users.

Enormous amount of data that generated by various IoT applications can be processed, stored and handled by cloud computing. However, the transmission of huge amount of data to/from cloud leads to a challenge because of limited bandwidth.

\section{FOG COMPUTING}

Fog computing can be seen as a layer between the underlying network and the cloud computing. Indeed, Fog computing extends the cloud computing to where the things are [24]. These devices, are called fog nodes. Fog computing is a platform, which provides computation, storage, and networking capabilities between the end devices and traditional cloud computing. Since number of connected devices are increasing, traditional cloud computing is not designed for the volume, variety, and velocity of data that the IoT devices generate. Analysis of big data generated by IoT devices, mostly must be realtime or near real-time. In order to attain this, data transmission, data storage and data processing must be performed in very short time intervals. Analyzing and storing IoT big data on devices which are physically close to the things, minimizes the latency. By this way, network traffic toward the core network can be reduced and realtime processing can be achieved. Also fog computing speeds up the sharpness and the response to events by analyzing data/event near to where it is generated [24]. These advantages can be met by following three parallel approaches:

- A considerable amount of data can be stored at or near the end-user (instead of storing whole data in remote data centers).

- A considerable amount of computing and control actions can be performed at or near the end-user (instead of doing all in remote data centers).

- A considerable amount of communication and networking can be carried out at or near the end- user (instead of routing all network traffic through the backbone networks).

Therefore, fog computing increases the overall performance of IoT applications as it performs a substantial part of high- level services inside the local resources and near the end-users rather than in the cloud. Of course, it cannot totally replace the cloud computing. It is obvious that, fog and cloud complement each other to build an adaptable and scalable platform for IoT. Fig 2. Presents architecture supporting IoT platform based on cloud and fog computing. In this architecture model, fog nodes do as following:

- Receive data/events from IoT heterogeneous devices in real-time using any protocol,

- Run IoT applications for real-time control, monitoring and analytics, with minimum response time,

- Provide temporary storage,

- Forward periodic data summaries to the cloud.

In a similar fashion, the cloud platform is responsible for:

- Aggregating data summaries from many fog nodes,

- Analyzing IoT data and data from other sources to gain business insight,

- Sending new application rules to the fog nodes based on these insights [24].

If IoT devices equipped with access network technologies such as 3G/4G/5G LTE, they would connect to internet directly. Otherwise, a smart gateway is required. Since some of the IoT devices are power-constrained, they use short-range wireless technologies such as Zigbee, BLE, IEEE 802.15.4 and Z-Wave to connect to gateway. These technologies have limited range up to a few hundred of meters [22]. Therefore, they are not suitable to connect low power devices distributed over large geographical areas such as a microgrid. A long range, low power, wide area and low bit rate wireless telecommunication system which is called LoRaWAN is used over a microgrid to connect devices into the gateway. IoT gateway can have two different approaches. First, it makes a simple connection between IoT devices and Internet. Second, it allows performing multiple operations (such as data refinement, filtering, 
trimming, and security measures) locally through fog computing and then to send important updates and necessary data to the cloud for synchronization [2]. Thus, real- time processing, low latency, bandwidth saving and traffic reduction are met.
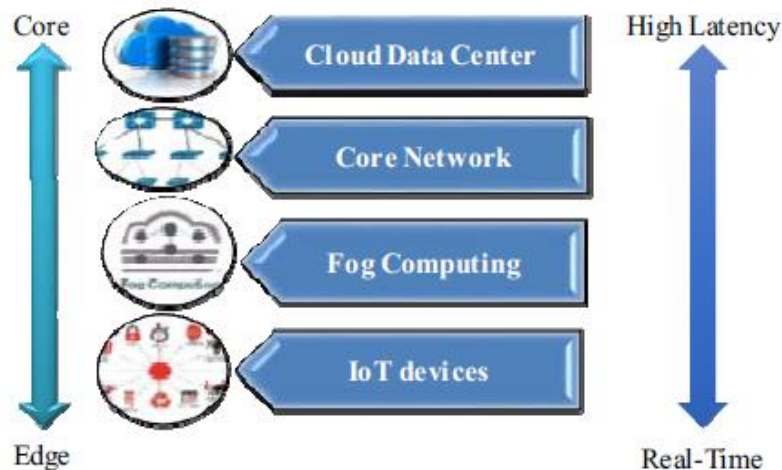

Real-Time

Figure 2 Architecture supporting IoT platform based on cloud and fog computing.

\section{LoRaWAN}

Low Power Wide Area (LPWA) networks expose a new communication model, which will supplement traditional cellular and short range wireless technologies to overcome varies requirement of IoT applications. LPWA is known by support for low power devices, with low data throughput requirements and long range operations. LoRaWAN is one of the LPWA technologies which designed to optimize range, energy consumption and bit rate in IoT applications. LoRaWAN is MAC protocol, build to use the LoRa physical layer [25]. LoRa is a physical layer technology that modulates the signals in SUBGHZ ISM band using a proprietary spread spectrum technique [26]. It provides a bidirectional communication by a special chirp spread spectrum (CSS) technique, which spreads a narrow band input signal over a wider channel bandwidth.

Technical specifications of LoRaWAN is described in table 1.

The long range and low power nature of LoRa makes it an interesting candidate for IoT and its application in smart grid.

A LoRaWAN network includes of end-devices, gateways and a server which collects and analyzes information extracted by the end-devices. The network topology is a "star of star topology", which means that groups of devices are connected to gateways via LoRa wireless links while the gateways are connected to a remote server via IP network (Fig 3). The server is located in a cloud [25].

LoRaWAN has three different classes of enddevices to address the various needs of applications:

Class A; bi-directional: This class of devices allows bidirectional communications, whereby each uplink transmission is followed by two short downlink receive windows. It provides the lowest energy consumption for end-devices, but this case has long delay in downlink.

- Class B, bi-directional with scheduled receive slots: Class B end-devices open extra receive windows at scheduled times. A synchronized beacon from the gateway is thus required, so that the network server is able to know when the end-device is listening.

- Class C, bi-directional with maximal receive slots: Class $\mathrm{C}$ end-devices have almost continuous receive windows. They thus have maximum power consumption.

Table 1. Technical specification of LoRaWAN

\begin{tabular}{|c|c|}
\hline Modulation & CSS \\
\hline Band & $\begin{array}{c}\text { SUB-GHZ ISM:EU } \\
\text { (433MHz } \\
\text { 868MHz), US (915MHz), } \\
\text { Asia } \\
(430 \mathrm{MHz})\end{array}$ \\
\hline Data rate & $\begin{array}{c}0.3-37.5 \text { kbps (LORa), } 50 \\
\text { kbps } \\
\text { (FSK) }\end{array}$ \\
\hline Range & $\begin{array}{c}5 \mathrm{~km} \text { (URBAN), } 15 \mathrm{~km} \\
\text { (RURAL) }\end{array}$ \\
\hline $\begin{array}{c}\text { Num. of } \\
\text { channels/orthogonal } \\
\text { signals }\end{array}$ & $\begin{array}{c}10 \text { in EU, } 64+8(\mathrm{UL}) \text { and } \\
\text { (DL) in } \\
\text { US plus multiple SFs }\end{array}$ \\
\hline Link symmetry & $\checkmark$ \\
\hline Forward error correction & $\checkmark$ \\
\hline MAC & unslotted ALOHA \\
\hline Topology & Star of stars \\
\hline Adaptive Data Rate & $\checkmark$ \\
\hline Payload length & $\begin{array}{c}\text { up to 250B (depends on SF } \\
\& \\
\text { region) }\end{array}$ \\
\hline Handover & $\begin{array}{c}\text { end devices do not join a } \\
\text { single } \\
\text { base station }\end{array}$ \\
\hline $\begin{array}{c}\text { Authentication \& } \\
\text { encryption }\end{array}$ & AES $128 b$ \\
\hline
\end{tabular}

\section{PROPOSED MODEL}

As mentioned previously, a smart grid is a smart energy hub which includes a variety of devices, including smart meters, smart appliances, renewable energy resources, etc. [27]. It can intelligently integrate the behaviors and actions of all users connected to it; including generators, consumers and prosumers, in order to efficiently deliver sustainable, economic and secure electricity supplies. It uses digital communications technology to detect and react to local changes in application. In addition, it 

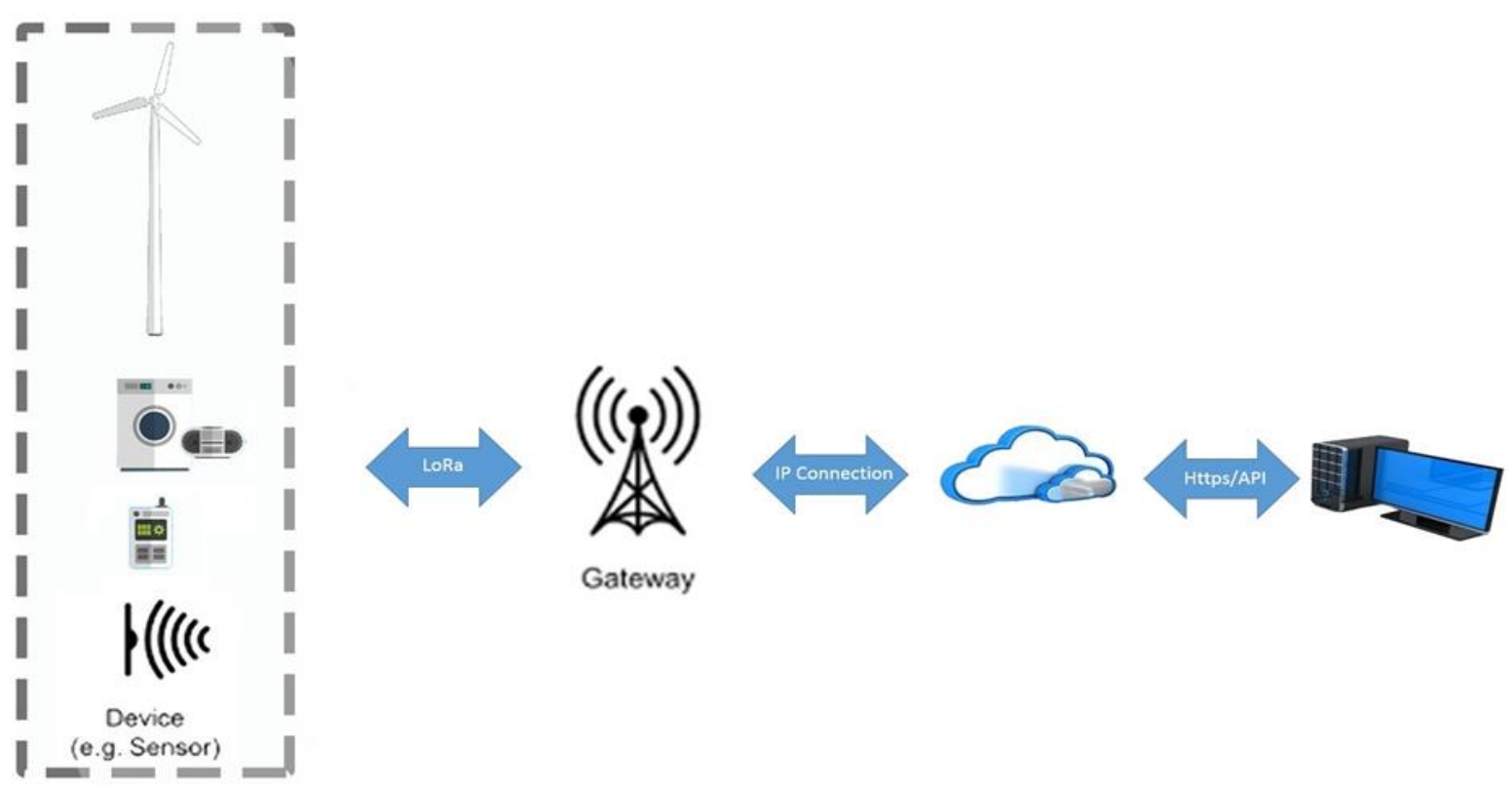

Gateway

Figure 3 LoRaWAN Topology.

enables demand side management through the integration of smart meters, smart appliances and consumers' loads, micro-generation units, energy storage options (electric vehicles) and by providing customers with information related to energy usage and prices. It is anticipated that customers will be provided with information and motivations to modify their consumption pattern to conquer some of the constraints in the power system. It also incorporates and comforts all renewable energy sources, distributed generation, residential microgeneration, and storage options [28].

Demand-side management is done by reducing the overall load on an electricity network. In fact, there are numerous connected devices to interact and cooperate with each other. The load shedding or load reduction is one of the demand side management approaches which is used in network scheduling and management phases. In

traditional networks, the load shedding program is determined as the priority of loads based on some predefined factors. In fact, this priority may be changed during different time intervals. However, in grid environment there are some uncertain parameters such as renewable energy resources which behave randomly during time. Therefore, it can affect the load shedding program in a way that the load decrement is changed according to the status of renewable energies in addition to the status of peak-load in the utility. In a microgrid with high penetration of renewable energy resources and local loads, the load shedding is determined mainly based on the priority of the loads and renewable energy status which can be changed dynamically. Hence, there are numerous data that should be transmitted and processed. However, to overcome increasing complexity and enormous amount of data generated by a large number of

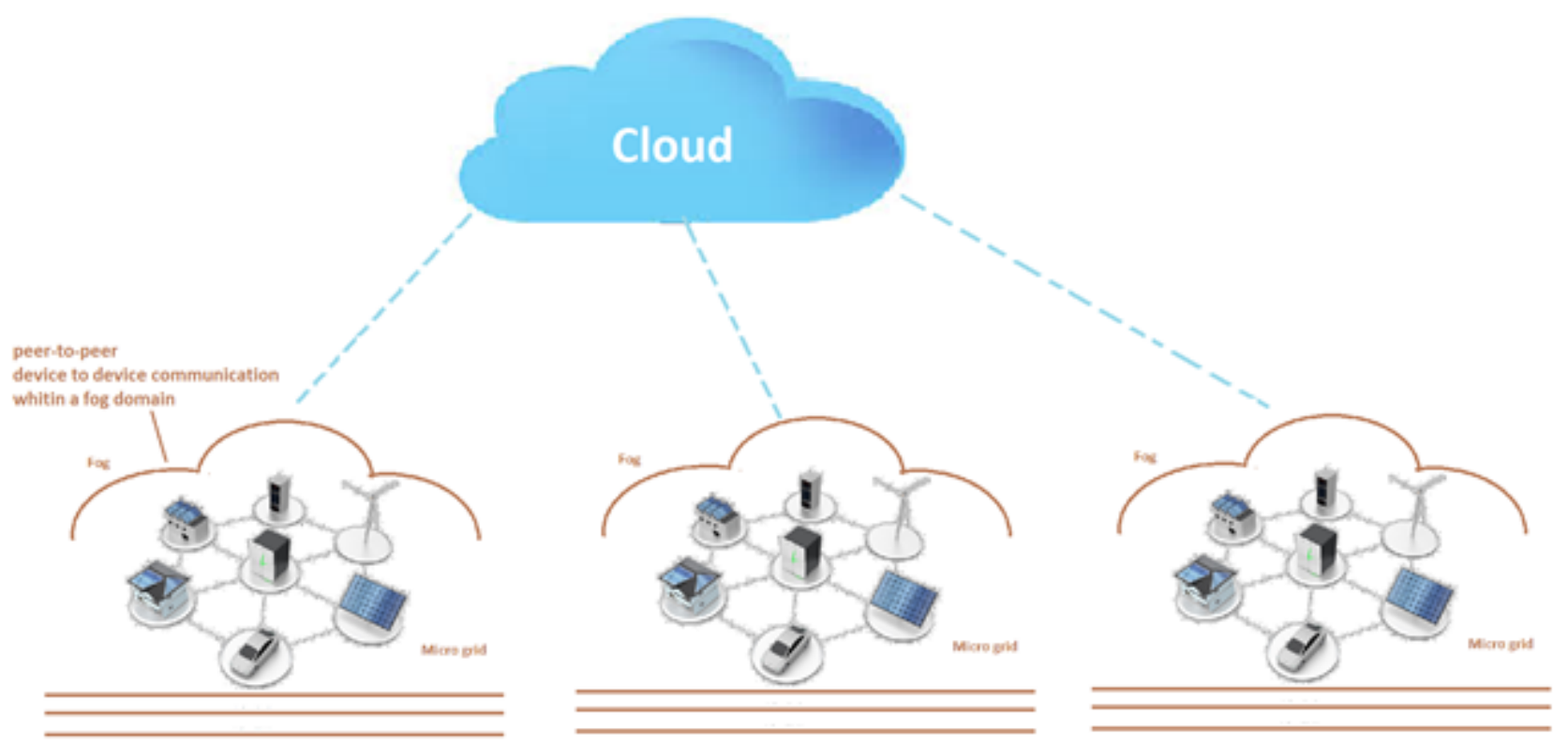

Figure 4 Microgrid considered as a fog domain. 


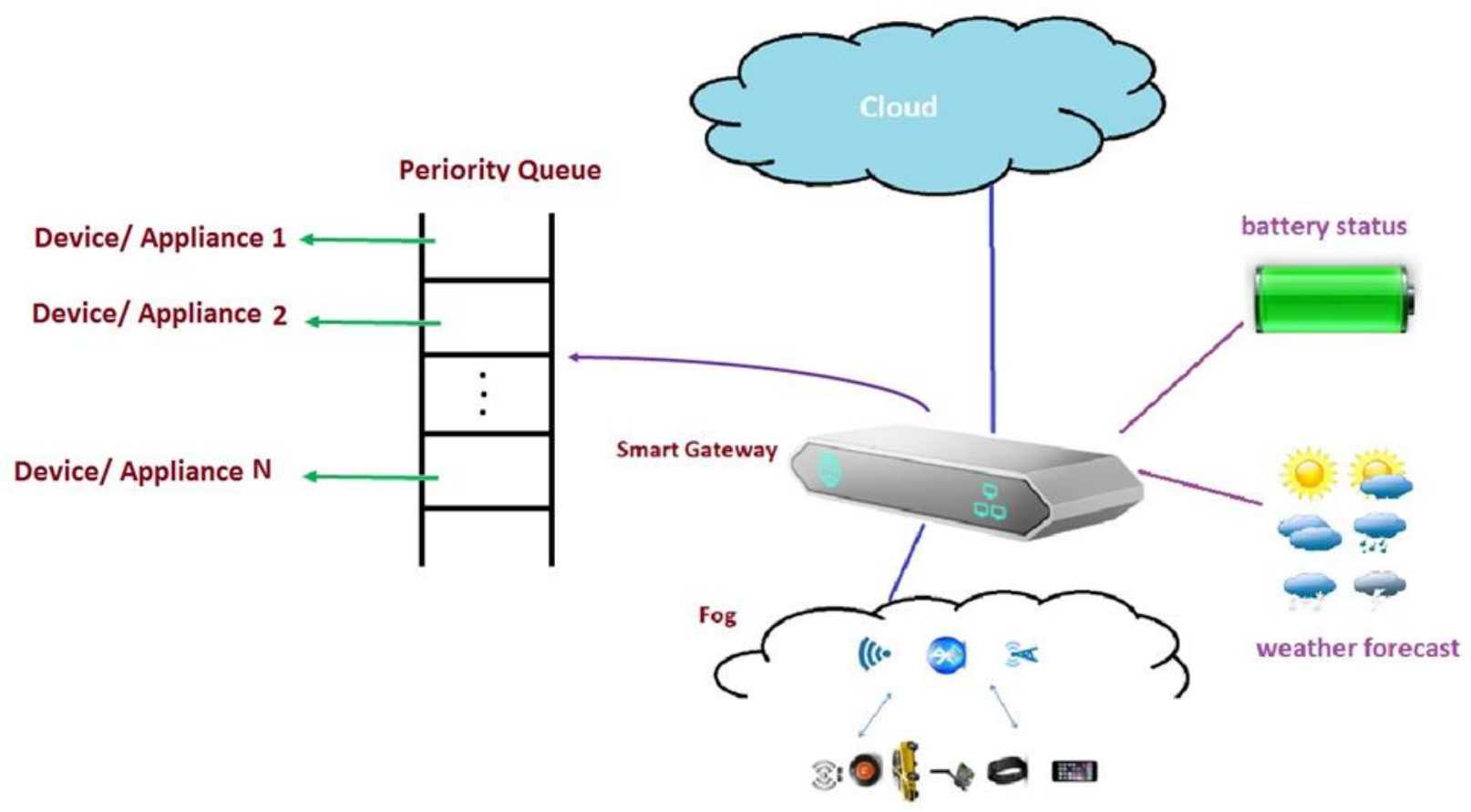

Figure 5 Smart gateway: point of connection between the fog and other components.

devices such as smart meters, sensors, actuators and relays, which has to be stored, processed, and accessed, the powerful processing resources are required which can be provide by cloud computing. The combination of cloud computing and IoT for creating IoE platform can enable ubiquitous sensing services, allowing the sensing data to be stored and used intelligently for smart monitoring and powerful processing of sensing data streams. At a same time, increasing the number of IoE devices leads to an increase in response time and latency in cloud computing, which causes deviations from the time requirements for some delay sensitive devices and applications. To conquer the cloud computing issues, the fog computing is suggested. The fog computing pulls the cloud computing to the edge of network and allows data to be preprocessed whenever latency limitation is required and leads to increase in interoperability, scalability, consistence and connection between smart devices. In a bulk electrical

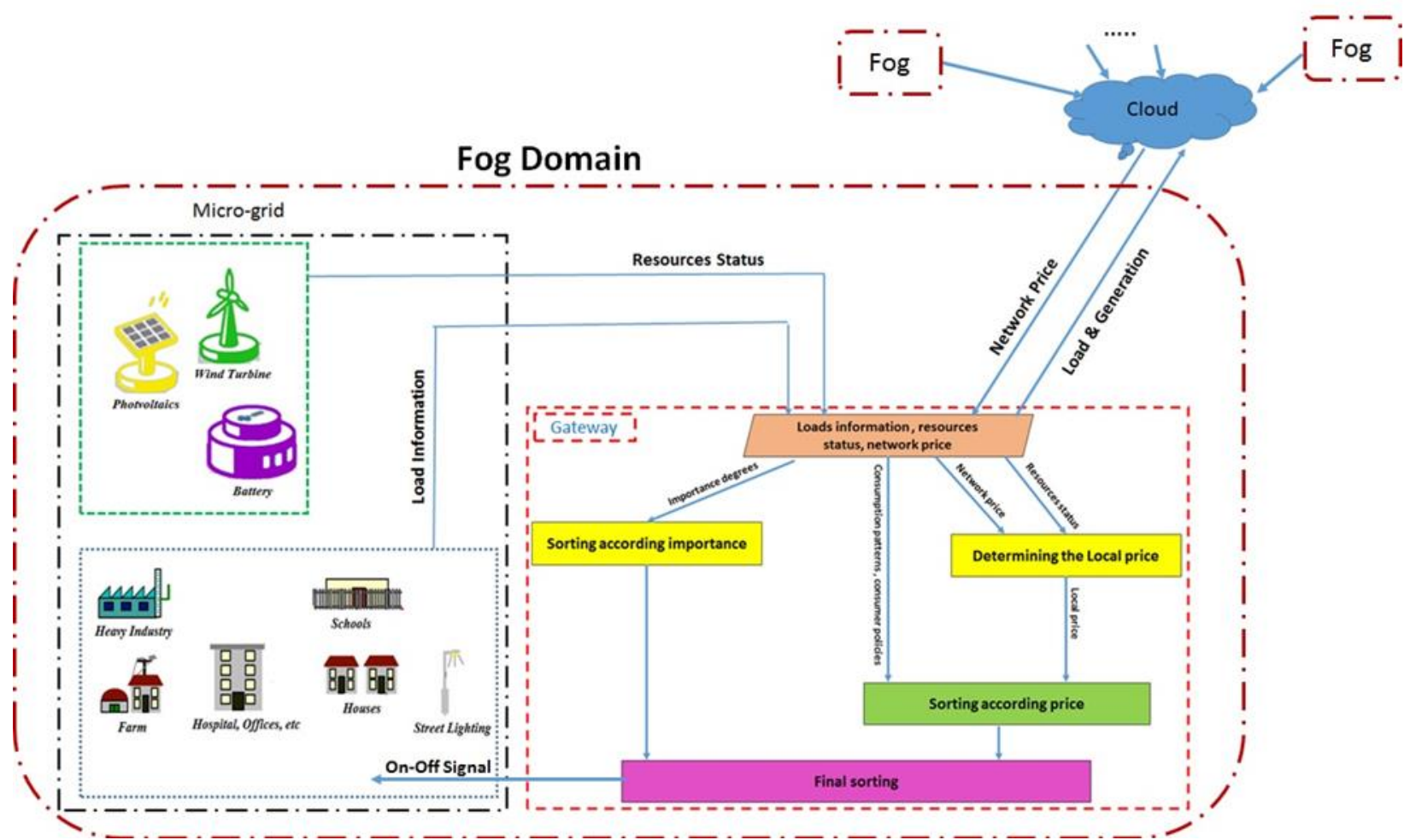

Figure $\overline{6}$ Queue sorting flowchart in a fog domain. 
network, the fog computing can be done on the local subnetworks, which are called microgrids. A microgrid is a small-scale local power grid that can operate independently or in connection with the utility grid, which constitutes of power resources, generation and loads and definable boundaries. Microgrid applications require realtime processing which can be carried out in the fog domain. Hence, data transmission rate into cloud, communication latency, traffic and bandwidth consumption are decreased. Since, in the proposed model it is tried to have the lowest data transmission from fog to cloud through core network, hence security and privacy are almost provided. As Fig. 4 shows, each microgrid considered as a fog domain and microgrid elements can communicate peer to peer to each other as well as to IoT gateway. In fact, smart gateway as shown in Fig. 5, bridges the fog and the cloud. Gateway as a fog node has computing, storage and networking capabilities. Moreover, two key components, local battery status and local weather forecasting, are connected to the gateway. As it can be seen in Fig. 5, there is a queue for microgrid which determined the priority of loads. The priority in queue can be changed dynamically based on the price and consumer's policies and importance. In addition, the status of renewable energies, which is predicted by weather forecast unit, can affect the priority in the queue and the amount of load shedding. Let's consider a scenario in a typical microgrid and in a peak-load situation where a priority queue can be made in gateway and scheduling can be done accordingly. In this case, gateway sends "off" or "reduce load" signals to lowest priority devices/ appliances. Therefore, due to local decision about load control, consumer's consumption turns economic. The queue sorting flowchart in fog domain is shown in Fig. 6. The load information, resources status and network price are the input data. The load information includes importance degree, consumption pattern and purchase policy of all loads. The purchase policy is defined according to local price by consumers. The local price is determined based on network price and the status of resources; e.g., in case of high price in network, if the renewable resources in microgrid can produce more, then the local price can be less than the network price. There are some important loads which should not be curtailed even in peak situation. Therefore, they will be at the beginning of the queue. The other loads approximately have the same importance degree which they can be curtailed. These loads would be sorted according to their purchase policies which are based on the price. These policies are announced by consumers to the gateway in each fog domain and they can be changed dynamically. Finally, the queue is sorted based on importance degrees and purchase policies momentary.

\section{CONCLUSION}

Load shedding or load reduction is one of the demand side management approaches which is used in network scheduling. In traditional networks, the load shedding program is determined as the priority of loads based on some predefined factors. In fact, this priority may be changed during time intervals. However, real grid environment there are some uncertain parameters such as renewable energy resources that have stochastic behavior during time. Therefore, it can affect the load shedding program in a way that the load decrement is changed according to the status of renewable energies in addition to the status of peak-load in the utility. In a microgrid, with high penetration of renewable energy resources and local loads, the load shedding is determined based on the priority of the loads and renewable energy status which can be changed dynamically. Hence, there are numerous data that should be transmitted and processed. In order to handle this multiplicity, the Internet can be used in electricity grid to monitor and control distributed loads. A large number of connected devices and the huge amount of data generated by IoT and issues related to transmit, process and storing it, force IoT to be integrated by cloud computing. Furthermore, in order to increase performance and reduce the volume of transmitted data and process data in acceptable time, fog computing is suggested as a layer between IoT layer and cloud layer. IoT devices equipped with access network technologies such as 3G/4G/5G LTE, they would connect to internet directly. Otherwise, a smart gateway is required. Since some of the IoT devices are power-constrained, and are distributed over large geographical areas such as a microgrid, therefore, a long range, low power, wide area and low bit rate wireless telecommunication system which is called LoRaWAN is used over a microgrid to connect devices into the gateway.

\section{REFERENCE}

[1] “Gartner's 2012 Hype Cycle for Emerging Technologies Identifies \& quot: Tipping Point \& quout; Technologies That Will Unlock LongAwaited Technologies Scenarios".

[2] F. Jalali, A. Vishwanath, J. De Hoog, and F. Suits, "Interconnecting Fog Computing and Microgrids for Greening IoT," pp. 0-5, 2016.

[3] L. Atzori, A. Iera, and G. Morabito, "The Internet of Things: A survey," Comput. Networks, vol. 54, no. 15, pp. 2787-2805, 2010.

[4] E. Borgia, "The Internet of Things vision: Key features, applications and open issues," Internet Things \Res. challenges Solut. vol. 54, pp. 1-31, 2014.

[5] "Bob Metcalfe's First PowerPoint Ever: The Ethernet Inventor Unveils the \&quot;Enernet\&quot; | Xconomy.".

[6] H. A. Gabbar, Smart energy grid engineering. 2017.

[7] World Energy Council, "World Energy Resources: 2013 survey," 2013.

[8] A. Anvari-Moghaddam, "Global Warming Mitigation Using Smart Micro-Grids," in Global Warming - Impacts and Future Perspective, B. R. Singh, Ed. INTECH, 2012, pp. 119-134.

[9] A. Q. Huang, M. L. Crow, G. T. Heydt, J. P. Zheng, and S. J. Dale, "The Future Renewable Electric Energy Delivery and Management (FREEDM) System: The Energy Internet," Proc. IEEE, vol. 99, no. 1, pp. 133-148, Jan. 2011. 
[10] G. Mokhtari, A. Anvari-Moghaddam, and G. Nourbakhsh, "Distributed Control and Management of Renewable Electric Energy Resources for Future Grid Requirements," in Energy Management of Distributed Generation Systems, 1st ed., no. July, Dr. Eng. Lucian Mihet, Ed. InT, 2016, pp. 1-24.

[11] P. Releases and P. Releases, "Renewables to lead world power market growth to 2020," 2015. [Online].Available:http://www.iea.org/newsroom/n ews/2015/october/renewables-to-lead-worldpower-market-growth-to-2020.html. [Accessed: 13Mar-2017].

[12] M. Parvizimosaed, F. Farmani, and A. AnvariMoghaddam, "Optimal energy management of a micro-grid with renewable energy resources and demand response," J. Renew. Sustain. Energy, vol. 5, no. 5, pp. 31-48, 2013.

[13] A. Anvari-Moghaddam, H. Monsef, A. RahimiKian, J. M. Guerrero, and J. C. Vasquez, "Optimized Energy Management of a Single-House Residential Micro-Grid With Automated Demand Response," in IEEE PES PowerTech Conference, 2015, pp. 1-6.

[14] M. Signorini, "Towards an Internet of Trust," 2015.

[15] A. Ghasemkhani, H. Monsef, A. Rahimi-Kian, and A. Anvari- Moghaddam, "Optimal Design of a Wide Area Measurement System for Improvement of Power Network Monitoring Using a Dynamic MultiObjective Shortest Path Algorithm," IEEE Syst. J., vol. PP, no. 99, pp. 1-12, 2015.

[16] P. Scott, "The emerging technologies," in California Management Review, vol. 45, no. April, 2005, pp. 2-6.

[17] M. A. Al Faruque and K. Vatanparvar, "Energy Management-as-a- Service over Fog Computing Platform," IEEE Internet Things J., vol. 3, no. 2, pp. 161-169, 2016.

[18] M. Aazam and E.-N. Huh, "Fog Computing and Smart Gateway Based Communication for Cloud of Things," in 2014 International Conference on Future Internet of Things and Cloud, 2014, pp. 464- 470.

[19] F. Bonomi, R. Milito, J. Zhu, and S. Addepalli, "Fog Computing and Its Role in the Internet of Things," Proc. first Ed. MCC Work. Mob. Cloud Comput. pp. 13-16, 2012.

[20] M. Chiang and T. Zhang, "Fog and IoT: An Overview of Research Opportunities," IEEE Internet Things J., vol. 4662, no. c, pp. 1-1, 2016.

[21] K. Shahryari, A. Anvari-Moghadam, "Demand side management using the internet of energy based on fog and cloud computing", International Conference on internet of things, and IEEE green computing and communications and IEEE Syber, 2017.

[22] U. Raza, P. Kulkami, M. Sooriabandara, "Low power wide area network: an overview", IEEE Communications Surveys \& Tutorials, vol. 19, no. 2, 2017, pp. 855-873.

[23] A. Rahman, M. Suryanegara, "The Development of IoT LoRa: A Performance Evaluation on LoS and Non-LoS Environment at 915MHz ISM Frequency
", International Conference of signals and systems, Sanur, Indonesia, 2017.

[24] Cisco Systems, "Fog Computing and the Internet of Things: Extend the Cloud to Where the Things Are," Www.Cisco.Com, p. 6, 2016.

[25] A. Augustin, J. Yi, T. Clausen, W. M. Townsley, “A study of LoRa: Long range \& Low power networks for the internet of things", Sensors, 16(9), 2016.

[26] F. Sforza, “Communications system," March 2013, uS Patent 8,406,275. [Online]. Available: https://www.google.com/patents/US8406275.

[27] A. Anvari-Moghaddam, AmjadSeifi, "A Comprehensive Study on Future Smart Grids: Definitions, Strategies and Recommendations," J. North Carolina Acad. Sci. vol. 127. No. 1, pp. 2834, 2011.

[28] J. Ekanayake, K. Liyanage, J. Wu, A. Yokoyama, and N. Jenkins, Smart Grid: Technologies and Applications. 2012. 